\title{
Modeling the interaction between ICM and relativistic plasma in cooling flows: The case of the Perseus cluster
}

\author{
M. Gitti ${ }^{1,2}$, G. Brunetti ${ }^{2}$, and G. Setti ${ }^{1,2}$ \\ 1 Dipartimento di Astronomia, via Ranzani 1, 40127 Bologna, Italy \\ 2 Istituto di Radioastronomia del CNR, via Gobetti 101, 40129 Bologna, Italy
}

Received 23 November 2001 / Accepted 14 February 2002

\begin{abstract}
We argue that the presence of diffuse synchrotron emission forming the so-called radio mini-halos in some cooling flow clusters can be explained by re-acceleration of relativistic electrons, the necessary energetics being supplied by the cooling flows themselves. In particular, the re-acceleration due to MHD turbulence has the correct radial dependence on the parameters to naturally balance the radiative losses. As an application we show that the main properties of the radio mini-halo in the Perseus cluster (brightness profile, total radio spectrum and radial spectral steepening) can be accounted for by the synchrotron radiation from relic relativistic electrons in the cluster, which are efficiently re-accelerated by MHD turbulence via Fermi-like processes due to the compression of the cluster magnetic field in the cooling flow region. Since the presence of an observable radio mini-halo in a cooling flow region critically depends on the combination of several physical parameters, we suggest that the rarity of radio mini-halos found in cooling flow clusters is due to the fact that the physical conditions of the ICM are intermediate between those which lead to the formation of extended radio halos and those holding in cooling flows without radio halos. The basic results of our model remain unchanged even if the cooling flow is stopped somewhere in the innermost region of the cluster.
\end{abstract}

Key words. acceleration of particles - radiation mechanisms: non-thermal - galaxies: cooling flows - galaxies: clusters: general - galaxies: clusters: individual: Perseus (A426)

\section{Introduction}

The intracluster medium (ICM) consists of a hot gas emitting thermal $\mathrm{X}$-rays, of large scale magnetic fields and of relativistic plasma. It is well known that due to synchrotron and inverse Compton (IC) losses, the typical ageing time-scale of the relativistic electrons in the ICM is relatively short $\left(10^{7} \div 10^{8}\right.$ yr $)$ so that the electrons should already have lost most of their energy. Nevertheless, diffuse synchrotron emission from clusters of galaxies has been detected by radio observations in a number of cases (e.g. Feretti 2000). Thus one can suppose that relativistic electrons may be re-accelerated by some mechanisms acting with an efficiency comparable to the energy loss processes (e.g. Petrosian 2001 and references therein) or, alternatively, one should turn to secondary electron models (Dennison 1980; Blasi \& Colafrancesco 1999; Dolag \& Enßlin 2000).

Large-scale radio halos in clusters of galaxies appear as diffuse radio sources of low surface brightness, steep

Send offprint requests to: M. Gitti, e-mail: mgitti@ira.cnr.it Present address: c/o Istituto di Radioastronomia del CNR, via Gobetti 101, 40129 Bologna, Italy radio spectrum and typical Mpc-size, not directly associated with the galaxies but rather diffused into the ICM at the center of the clusters (Feretti \& Giovannini 1996 and references therein). Liang et al. (2000) have shown the presence of a correlation between radio power and cluster temperature for radio halos with good radio and X-ray data available. This correlation, together with the morphological similarity of thermal X-rays and radio emission (Govoni et al. 2001), suggests a physical relationship between the properties of thermal ICM and the presence of radio halos. It is also well known that there exists an anti-correlation between the presence of a cooling flow and of a radio halo at the cluster center: radio halos are rarely found in clusters of galaxies with cooling flow and, when they are, they appear quite different from canonical halos such as that in the Coma cluster (Tribble 1993). However, there are a few cooling flow clusters where the relativistic particles can be traced out quite far, forming what is called a "mini-halo": a radio source smaller in extent, of low surface brightness and steep spectral index, around a powerful radiogalaxy at the center of a cluster (e.g. Virgo: Owen et al. 2000; Perseus: Burns et al. 1992; PKS 0745-191: Baum \& O’Dea 1991; A2626: Rizza et al. 2000). 
On the basis of the above considerations, it is clear that the persistent diffuse radio emission, and hence the need for re-acceleration of the relativistic plasma, may be found in the interaction between the thermal and the relativistic component in the ICM. Indeed, Brunetti et al. (2001) have proposed that the relativistic electrons injected in the cluster volume by AGN and/or galactic winds in the course of the cluster evolution may form a relic electron population and that an extended radio halo is originated if the relic, but still relativistic, electrons are re-accelerated to higher energies by shocks and/or turbulence in the ICM. In this model, as suggested by observations, the merger events may supply the necessary energy for the re-acceleration. In this paper we argue that a similar model holds for the relativistic electrons in the mini-halos too, and that the necessary energetics is supplied by the cooling flows, as suggested by Tribble (1993) and Sijbring (1993). Unlike the case of the extended radio halos, the energy can not arise from the merger events, since observations in general show no mergers acting in clusters with cooling flows (Edge et al. 1992).

The main aim of the present work is to investigate whether the re-acceleration of relic electrons and the increased intensity of the cluster magnetic field due to the compression in the turbulent cooling flow can produce radio mini-halos. The model expectations are then compared with the observational properties of the radio emission from the mini-halo in the Perseus cluster, where a massive cooling flow is present (Ettori et al. 1998).

In Sect. 2 we consider the effect of a cooling flow on the intracluster magnetic field and calculate the electron energy distribution subject to re-acceleration and losses. In Sect. 3 we present the results concerning the radio properties of the radio mini-halo in the Perseus cluster. Our conclusions are given in Sect. 4 .

$H_{0}=50 \mathrm{~km} \mathrm{~s}^{-1} \mathrm{Mpc}^{-1}$ is assumed in this paper and, where not specified, all the formulae are in cgs system.

\section{The models for electron re-acceleration}

\subsection{Magnetic fields and relativistic electrons in cooling flows}

X-ray and optical observations indicate that large amounts of gas are cooling and flowing into the centers of clusters of galaxies; the observations and theory of "cooling flows" are reviewed in Fabian (1994). For thermal bremsstrahlung models, the decrease of the temperature with the distance $r$ from the center is $T(r) \propto r^{1.2}$, whereas the gas density increases towards the center with the same behaviour (Fabian et al. 1984). The compression of the gas is expected to produce a sensible increase of the strength of the frozen-in intracluster magnetic field. Depending on the physical conditions prevailing in a cooling flow, in particular on the values of the turbulent velocity $v_{\mathrm{T}}$ and of the mean inflow velocity $v_{\mathrm{F}}$, there are two possibilities:

- if $v_{\mathrm{T}} \ll v_{\mathrm{F}}$, the inward cooling flow is homogeneous and the turbulence does not distort the field geometry

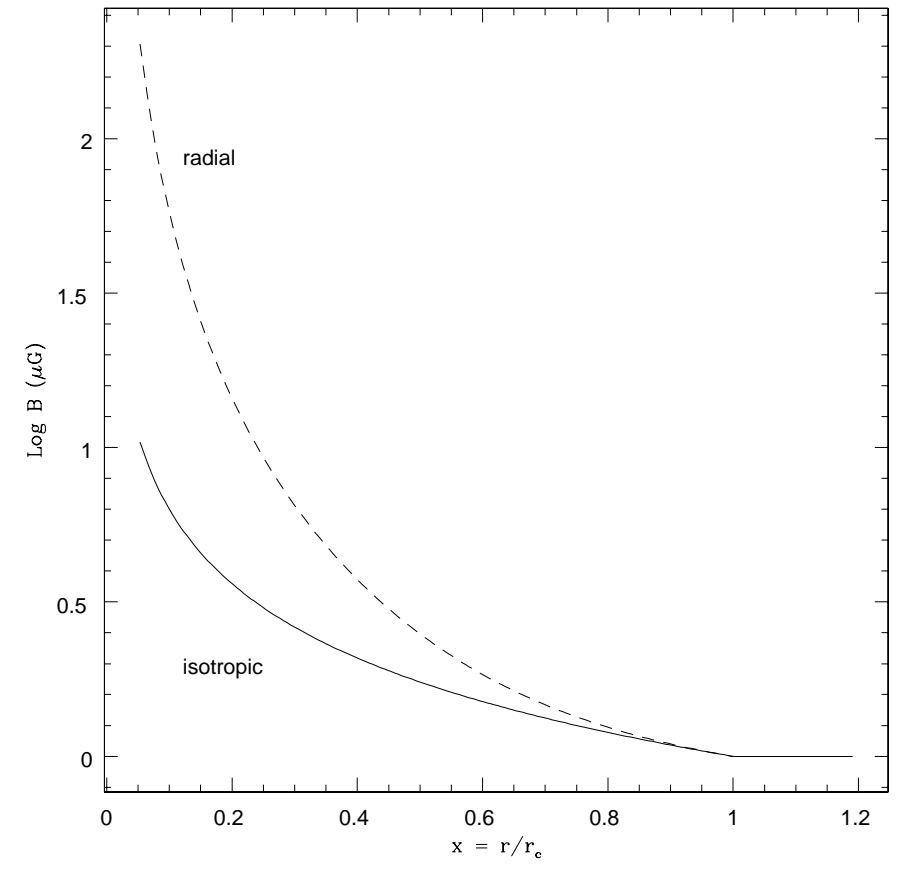

Fig. 1. The calculated field growth in the cooling flow region for Soker \& Sarazin's model (radial field, dashed line) and Tribble's model (isotropic field, solid line). We have assumed $r_{\mathrm{c}}=210 \mathrm{kpc}$ and $B_{\mathrm{c}}=1 \mu \mathrm{G}$.

during the infall. In this case we can follow Soker \& Sarazin's model (1990). They have presented numerical solutions showing that the frozen-in magnetic field is greatly increased by the compression of the ICM and its lines become predominantly radial as the gas flows inward. Under the assumption that the field is not dynamically important, one has that

$B(x)=\frac{B_{\mathrm{c}}}{\sqrt{3}} x^{-2}\left(1+2 x^{\frac{18}{5}}\right)^{\frac{1}{2}} \sim x^{-2}$

where $r_{\mathrm{c}}$ is the cooling radius, $x=r / r_{\mathrm{c}}$ is the adimensional distance from the center and $B_{\mathrm{c}}$ is the field strength at $r_{\mathrm{c}}$.

- if $v_{\mathrm{T}} \gg v_{\mathrm{F}}$, as pointed out by Tribble (1993), an individual element of gas in the flow is in constant motion relative to the mean flow and its mean position falls slowly inwards. The overall effect is an isotropic compression of the field. In this case the variation of the field with the gas density $n$ within the central cooling region is $B \propto n^{\frac{2}{3}}$, so that being $n(x) \propto x^{-1.2}$, it results

$$
B(x)=B_{\mathrm{c}} x^{-0.8} \text {. }
$$

The resulting intensity profile of the magnetic field in these two different cases is plotted in Fig. 1. In both cases the field strength grows towards the center, but with quite different behaviours. In Soker \& Sarazin's model the field strength grows faster than in Tribble's one and in the central region the field reaches values which are at least one order of magnitude larger than those reached by the isotropic compression. It should be mentioned that intense 
fields of the order of some tens of $\mu \mathrm{G}$ have been derived by detections of extremely high Faraday rotation measures throughout radio galaxies in the center of cooling flow clusters (Taylor \& Perley 1993; Ge \& Owen 1993; Taylor et al. 1990; Perley \& Taylor 1991). This has important consequences on the evolution of the relativistic electron energy and spectrum. Indeed, the radiative lifetime of an ensemble of relativistic electrons losing energy by synchrotron emission and IC scattering off the cosmic microwave background photons $(\mathrm{CMB})$ is given by

$\tau_{\sin +I C}=\frac{16.2}{\left[B_{\perp}^{2}+\frac{2}{3} B_{\mathrm{CMB}}^{2}\right] \gamma} \mathrm{yr}$

where $\gamma$ is the Lorentz factor and $B_{\mathrm{CMB}}=3.18(1+z)^{2} \mu \mathrm{G}$ denotes the magnitude of the magnetic field equivalent to the CMB from the standpoint of the losses. Without any re-acceleration mechanism at work, the emission of these electrons in the radio band should not be observable for more than $\sim 10^{8} \mathrm{yr}$. This short lifetime contrasts with the diffuse radio emission observed, for example, in the Perseus cluster: whatever the model for the origin and presence of the relativistic electrons in the mini-halo region is, this characteristic lifetime is both shorter than the crossing time (both assuming the Alfvén velocity or the diffusion coefficient as in Sarazin 2001), and shorter than the cooling time (if one assumes that the radio-emitting region is filled with electrons carried by the cooling flow). Hence, it seems plausible that the electrons have been reaccelerated.

A re-accelerated relic electron population in cluster radio halos would naturally produce radio synchrotron spectra which steepen with increasing distance from the center (as observed in the Coma radio halo), whereas it cannot be produced in the case of models involving a secondary electron population (Brunetti et al. 2001). Here we stress that the radio spectral index map of the mini-halo in the Perseus cluster shows a progressive steepening of the spectral index with increasing distance (Sijbring 1993), thus providing an additional indication for the presence of an effective mechanism for electron re-acceleration operating in the ICM in the cooling flow region.

\subsection{Evolution of electron energy spectrum into the cooling flow}

In our model we assume that the relativistic electrons are continuosly re-accelerated by Fermi type mechanisms. Given an initial monoenergetic electron distribution, the electrons are systematically accelerated with an efficiency $\alpha_{2}$ and, at the same time, their energy is stocastically changed with an efficiency $\alpha_{1}$. These two combined effects result in a relatively wide distribution of the energy of the electrons around a mean value determined by systematic processes. In particular, it is well known that MHD turbulence can efficiently accelerate electrons via Fermi-like processes. In order to allow relativistic electrons to still be emitting in the radio band, the re-acceleration mechanism has to act with an efficiency comparable to the energy loss processes. Due to the competition of these two effects, at any distance $x$ the time evolution of the energy of a relativistic electron is given by:

$\frac{\mathrm{d} \gamma(x)}{\mathrm{d} t}=-\beta(x) \gamma^{2}(x)+\alpha_{2}(x) \gamma(x)-\chi(x)$

where $\beta(x)$ is the coefficient of synchrotron and IC losses, $\alpha_{2}(x)$ the acceleration coefficient and $\chi(x)$ the Coulomb losses term. The Coulomb losses depend on the ICM density as (e.g. Sarazin 1999):

$\chi(x) \sim 1.4 \times 10^{-12} n(x) \quad \mathrm{s}^{-1}$

where $n(x)=n_{\mathrm{c}} x^{-1.2}, n_{\mathrm{c}}$ being the electron density at the cooling radius.

The coefficient of the radiative losses is:

$\beta(\vartheta, x)=1.9 \times 10^{-9}\left[B^{2}(x) \sin ^{2} \vartheta+\frac{2}{3} B_{\mathrm{CMB}}^{2}\right] \quad \mathrm{s}^{-1}$

where $\vartheta$ is the pitch angle and where we have neglected the dependence on the redshift, not important in our model. If the pitch angle scattering is an efficient process, then the electrons will be continuosly isotropized and the term $B^{2}(x) \sin ^{2} \vartheta$ should be replaced by $2 / 3 B^{2}(x)$, where $B(x)$ is obtained from Eq. (1) or (2).

According to the usual Fermi acceleration theory (e.g. Melrose 1980), it is $\alpha_{2}=2 \alpha_{1}$ and the coefficient of systematic Fermi acceleration due to MHD turbulence is given by:

$\alpha_{2}(x) \sim \frac{v_{\mathrm{A}}^{2}(x)}{l(x) c}\left(\frac{\delta B(x)}{B(x)}\right)^{2}$

where $v_{\mathrm{A}}(x) \sim 2.2 \times 10^{11} n_{\mathrm{c}}^{-0.5} x^{0.6} B(x) \quad \mathrm{cm} \mathrm{s}^{-1}$, and $l(x)$ is the characteristic distance between two subsequent peaks of magnetohydrodynamic turbulence.

We assume the existence of an energetic turbulence such that $\delta B(x) / B(x) \sim 1$ constant through the whole cooling flow region. Since the characteristic time of radiative losses depend on $\gamma$, while that of Fermi acceleration is independent of $\gamma$, the losses dominate the time evolution of the electrons for energies higher than $\gamma_{\mathrm{b}}$, the break energy. It is worth noticing that MHD turbulence can efficiently accelerate electrons via Fermi-like processes when their velocity parallel to the magnetic field is $v_{\|}>\left(m_{\mathrm{p}} /\left(m_{\mathrm{e}} \mu\right)\right)^{1 / 2} \times v_{\mathrm{A}}$, where $v_{\|}=v \mu($ Eilek \& Hughes 1991; Hamilton \& Petrosian 1992). The fact that in our model we deal with a seed population of relativistic electrons guarantees the efficiency of this process. Under the simple assumption that the total number of turbulent elements is conserved during the infall, $l(x)$ is roughly proportional to $n(x)^{-\frac{1}{3}}$, so that $l(x)=l_{\mathrm{c}} x^{0.4}, l_{\mathrm{c}}$ denoting the characteristic length at the cooling radius. The underlying assumption is that the turbulence does not decay on a time scale shorter than the acceleration time scale.

By solving Eq. (4) at any time $t$, one can calculate the break energy $\gamma_{\mathrm{b}}(t)$, corresponding to the energy at the time $t$ of the electrons having a near infinite energy at 
the initial time $t=0$. By omitting for simplicity of notation the dependence on the distance from the cluster center, it results (Brunetti et al. 2001):

$\gamma_{\mathrm{b}}(t)=\frac{1}{2 \beta}\left(\alpha_{2}+\frac{\sqrt{\alpha_{2}^{2}-4 \beta \chi}}{\tanh \left(\frac{t}{2} \sqrt{\alpha_{2}^{2}-4 \beta \chi}\right)}\right)$.

The break energy of the stationary spectrum, which corresponds to the energy at which the losses are balanced by the re-acceleration, is obtained by evaluating Eq. (8) in the limit $t \rightarrow \infty$ :

$\gamma_{\mathrm{b}}=\frac{1}{2 \beta}\left(\alpha_{2}+\sqrt{\alpha_{2}^{2}-4 \beta \chi}\right)$.

Because of the distance dependence of the coefficients, the break energy in the electron spectrum is not the same throughout the whole cooling flow region, but it (weakly) depends on the radial distance.

The time evolution of the electron energy distribution is obtained by solving the continuity equation (Kardashev 1962) taking into account the acceleration by the Fermi mechanism and the losses.

Since the acceleration time scale (Eq. (7))

$\tau_{\text {acc }}=\alpha_{2}^{-1} \sim 6 \times 10^{7} l(\mathrm{pc})\left(\frac{n}{10^{-3}}\right) B^{-2}(\mu \mathrm{G}) \quad \mathrm{yr}$

(e.g. $\tau_{\text {acc }} \sim 8 \times 10^{8} \mathrm{yr}$ for the values of parameters obtained for the Perseus cluster at the cooling radius, Sect. 3) than the time it takes for a volume element to be convected by the flow from $r_{\mathrm{c}}$ into the center, we can solve the continuity equation under stationary conditions, obtaining (for $\gamma \gg 1)$

$N(\gamma)=N\left(\gamma_{\mathrm{b}}\right) \exp (\Phi) \cdot\left(\frac{\gamma}{\gamma_{\mathrm{b}}}\right)^{2} \exp \left[f\left(\frac{\gamma}{\gamma_{\mathrm{b}}}\right)\right]$

where $N\left(\gamma_{\mathrm{b}}\right)$ is the number of electrons at $\gamma_{\mathrm{b}}$, $\Phi=2-\frac{4}{1+\frac{x}{\beta \gamma_{\mathrm{b}}{ }^{2}}}$ and

$f\left(\frac{\gamma}{\gamma_{\mathrm{b}}}\right)=\frac{\frac{2 \chi}{\gamma_{\mathrm{b}}^{2}}-2 \beta\left(\frac{\gamma}{\gamma_{\mathrm{b}}}\right)^{2}}{\left(\beta+\frac{\chi}{\gamma_{\mathrm{b}}}\right)\left(\frac{\gamma}{\gamma_{\mathrm{b}}}\right)}$.

Since in our case it is $\chi /\left(\beta \gamma_{\mathrm{b}}^{2}\right) \ll 1$ and $\chi /\left(\beta \gamma_{\mathrm{b}}^{2}\right) \ll$ $\left(\gamma / \gamma_{\mathrm{b}}\right)^{2}$, Eq. (11) can be approximated as $N(\gamma) \propto$ $\left(\gamma / \gamma_{\mathrm{b}}\right)^{2} \exp \left(-2 \gamma / \gamma_{\mathrm{b}}\right)$, in agreement with the result of Borovsky \& Eilek's (1986), that indeed have not considered the Coulomb losses.

Note that the spectral distribution of the electrons $N(\gamma)$ (Eq. (11)) depends on $\gamma / \gamma_{\mathrm{b}}$ only, while the values of the coefficients simply produce a "normalisation" of the spectrum which depends on the radial distance.

In principle, in order to correctly derive the energy density of the relativistic electrons as a function of $x$ one should solve the spatial diffusion equation for the relativistic electrons starting from some initial distribution of the number density and spectrum of the electrons which, however, are basically unknown. As a consequence, in this paper we prefer to parameterize the electron energy density, essentially peaked at $\gamma \sim \gamma_{\mathrm{b}}$, as:

$m_{\mathrm{e}} c^{2} \int_{1}^{\infty} N(\gamma) \gamma \mathrm{d} \gamma \approx m_{\mathrm{e}} c^{2} N\left(\gamma_{\mathrm{b}}\right) \gamma_{\mathrm{b}}^{2} \propto x^{-s}$

where $s$ is a free parameter which will be constrained and discussed in Sect. 3 .

\subsection{The synchrotron spectrum}

The synchrotron emissivity of a population of relativistic electrons with energy distribution per unit solid angle $N(\gamma, \Omega)$ is given by:

$J_{\nu}=\int_{\Omega} \int_{1}^{\infty} p(\nu, \vartheta) N(\gamma, \Omega) \mathrm{d} \gamma \mathrm{d} \Omega$

where $p(\nu, \theta)=\frac{\sqrt{3} e^{3} B \sin \vartheta}{m_{\mathrm{e}} c^{2}} F\left(\frac{\nu}{\nu_{\mathrm{c}}}\right)$ is the emitted power per unit frequency and per unit solid angle from each electron, $F\left(\frac{\nu}{\nu_{\mathrm{c}}}\right)$ being the Kernel function and $\nu_{\mathrm{c}}=\frac{3}{4 \pi} \frac{e B \sin \vartheta}{m_{\mathrm{e}} c} \gamma^{2}$ the critical frequency (Ginzburg \& Syrovatskii 1965). Under the assumption of an isotropic distribution of electron momenta and of the magnetic field lines we can assume the synchrotron emissivity to be isotropic, if averaged over a sufficiently large volume.

With the energy distribution of the relativistic electrons given by Eq. (11), the synchrotron emissivity per unit solid angle is:

$$
\begin{aligned}
J_{\nu}(x) & =K(x) \cdot \int_{0}^{\frac{\pi}{2}} \int_{1}^{\infty} \sin ^{2} \vartheta \cdot F\left(\frac{\nu}{\nu_{\mathrm{b}}} \frac{\gamma_{\mathrm{b}}^{2}}{\gamma^{2} \sin ^{2} \vartheta}\right) \cdot \gamma^{2} \\
& \cdot \exp \left[f\left(\frac{\gamma}{\gamma_{\mathrm{b}}}\right)\right] \mathrm{d} \vartheta \mathrm{d} \gamma
\end{aligned}
$$

where

$K(x)=\frac{\sqrt{3} e^{3}}{m_{\mathrm{e}} c^{2}} \cdot B(x) \cdot N\left(\gamma_{\mathrm{b}}\right)_{\mathrm{c}} \frac{\gamma_{\mathrm{b}, \mathrm{c}}{ }^{2}}{\gamma_{\mathrm{b}}{ }^{4}} \cdot \exp (\Phi)$

$\nu_{\mathrm{b}}$ being the critical frequency for $\gamma=\gamma_{\mathrm{b}}\left(\vartheta=90^{\circ}\right)$. The shape of the synchrotron spectrum is plotted in Fig. 2: at low frequencies the synchrotron spectrum is approximately proportional to $\nu^{-1 / 3}$, as in the case of the emission by a single electron, it becomes roughly flat just below the break frequency (contributed by the electrons at the break energy $\gamma_{\mathrm{b}}$ ) and then it falls down exponentially.

\section{Model results for the Perseus cluster}

The Perseus cluster (Abell426), at a redshift $z=0.0183$, is one of the brightest clusters in the sky in X-ray band and hosts the nearest large cooling flow. $\mathrm{X}$-ray deprojection analysis of ROSAT PSPC surface brightness profiles indicates that the mass deposition rate is about $500 M_{\odot} \mathrm{yr}^{-1}$ and the cooling radius is about $210 \mathrm{kpc}$ (Ettori et al. 1998); with these values of $\dot{M}$ and $r_{\text {c }}$, one estimates $n_{\mathrm{c}} \sim 1.2 \times 10^{-3} \mathrm{~cm}^{-3}$. The dominant galaxy in the cluster core is the active galaxy NGC 1275 . The associated radio source (Perseus A or $3 \mathrm{C} 84$ ) is one of the brightest radio 


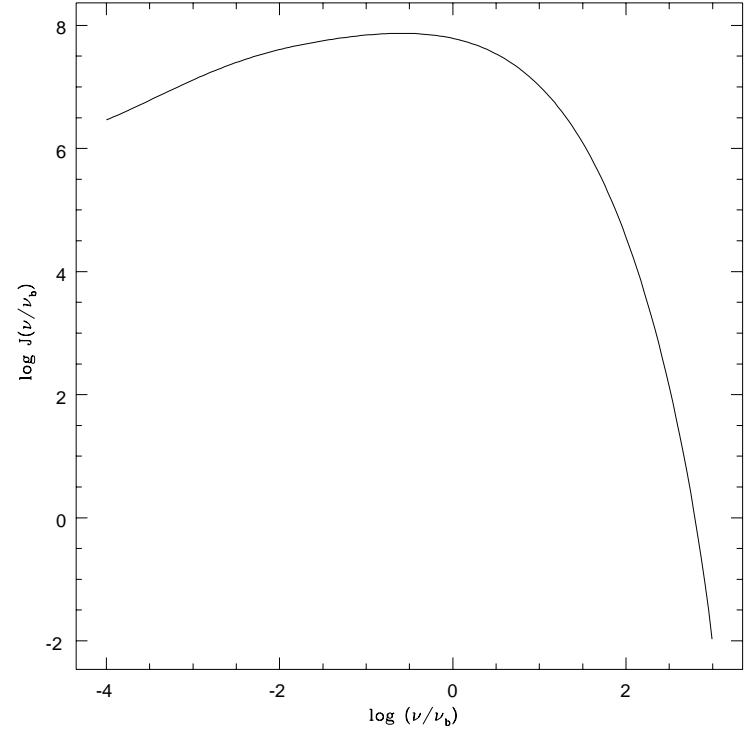

Fig. 2. The predicted synchrotron spectrum is shown in arbitrary units.

sources (the total flux density at $327 \mathrm{MHz}$ is $40.66 \mathrm{Jy}$ ), with a complex structure and a mini-halo whose extension $\left(\sim 15^{\prime}\right)$ is comparable with that of the cooling flow region. The radio mini-halo has a steep spectrum with spectral index $\alpha=1.4$ (between $327 \mathrm{MHz}$ and $609 \mathrm{MHz}$, $F_{\nu} \propto \nu^{-\alpha}$ ) and its total flux is $17.57 \mathrm{Jy}$ at $327 \mathrm{MHz}$; in addition there is evidence for a spectral steepening in the outer regions (Sijbring 1993).

Because of the simultaneous presence of the radio mini-halo and of the cooling flow, we can apply to the Perseus cluster the model for electron re-acceleration presented in the previous section. For comparison with the model expectations we have considered the $92 \mathrm{~cm}$ $(327 \mathrm{MHz})$ radio image shown in Fig. 3. In this map the bright radio core of $3 \mathrm{C} 84$ can be very well approximated by a point-like source and we have estimated that the diffuse mini-halo emission is affected by this intense central emission only within $\sim 1^{\prime}$ from the cluster center, in agreement with ROSAT and Chandra imaging which show an interaction between the radio lobes of $3 \mathrm{C} 84$ and the ICM only on this scale (Böringer et al. 1993; Fabian et al. $2000)$. For this reason, our model should be applicable for $r \gtrsim 30 \mathrm{kpc}$.

Figure 3 shows that the diffuse mini-halo is rather asymmetric so that, because of the spherical symmetry assumed in our model, in order to compare the expected and observed radio emission we have considered a particular direction across the apparently more regular region deprived of field radio sources. However, it is worth noticing that a strong morphological correlation has been shown to hold between the cooling flow X-ray map and the radio mini-halo (Ettori et al. 1998; Sijbring 1993). This means that our choice of a specific direction does not appear as an effective restriction to the conclusions we are led to by the application of our spherically symmmetric model to such an asymmetric object. On the contrary, our results could

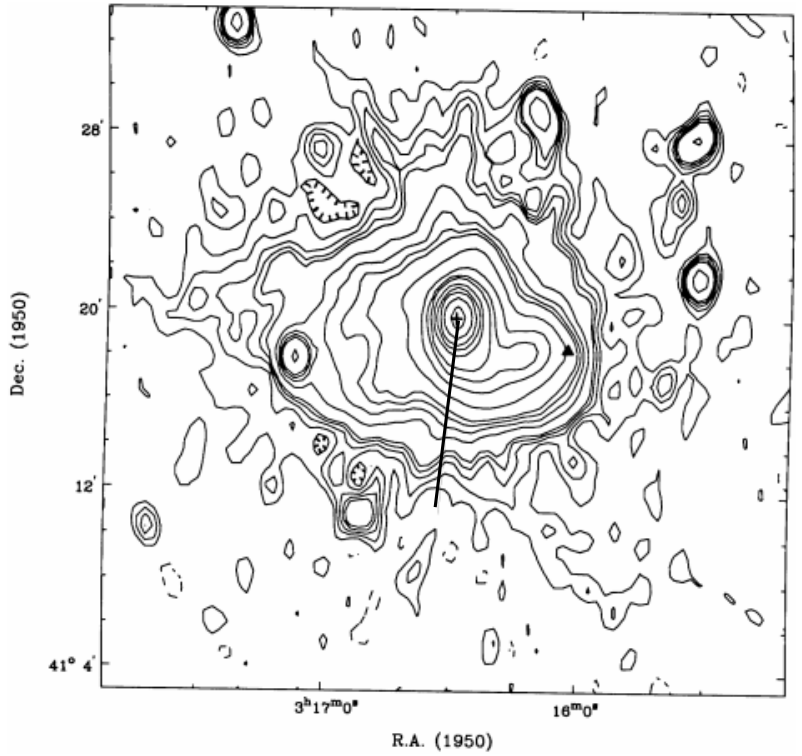

Fig. 3. $92 \mathrm{~cm}$ map of $3 \mathrm{C} 84$ at a resolution of $51^{\prime \prime} \times 77^{\prime \prime}$ (Sijbring 1993). The contour levels are -4 (dashed), 4, 8, 12, 16, 20, $40,60,80,100,200,400,600,800,1500,2500,4000,8000$, $16000 \mathrm{mJy} /$ beam. The rms noise is $1.4 \mathrm{mJy} /$ beam. The cross indicates the position of NGC1275 and the solid line represents the direction we have considered.

reasonably be applied to any other direction, once the correct values for the physical parameters of the cooling flow in that particular direction are taken into account (Ettori et al. 1998). Moreover, this strong morphological correlation can be taken as a hint to the influence of the cooling flow over the relativistic plasma behaviour, hence supporting the idea of the electron reaccleration being powered by the cooling flow itself.

In order to compare the model with the observations, the observational constraints are:

brightness profile: we have calculated the surface brightness profile expected by our model by integrating the synchrotron emissivity (Eq. (15)) along the line of sight;

total spectrum: the total synchrotron spectrum is obtained by integrating the synchrotron emissivity over the cluster volume taking into account the $x$-dependence of the parameters involved in the calculation;

radial spectral steepening: at each distance $x$ we obtain from Eq. (15) the synchrotron emissivity as a function of the frequency measured in terms of the break frequency and then compute the spectral index between $327 \mathrm{MHz}$ and $609 \mathrm{MHz}$.

We have separately considered the two cases of field compression previously discussed (see Sect. 2.1).

The free parameters of our model are the energy density distribution of the relativistic electrons, i.e. the parameter $s$ of Eq. (13), and the values at the cooling radius of the magnetic field intensity, $B_{\mathrm{c}}$, and of the characteristic distance between two subsequent peaks of magnetohydrodynamic turbulence, $l_{\mathrm{c}}$. In order to investigate the range of parameters required by the model to match the observations, the calculation has been performed as 
follows: for each case of field compression we have fixed $s$ and we have allowed the other two parameters $B_{\mathrm{c}}$ and $l_{\mathrm{c}}$ to vary; then, for a given set of parameters, we have calculated the brightness profile, the total spectrum and the radial spectral steepening and compared them with the observations.

Tribble's case of isotropic compression of the field appears to be very promising. The best results are obtained for $1.5 \lesssim s \lesssim 2.5$. The parameter space which well reproduces the surface brightness profile and the total synchrotron spectrum is in good agreement with that producing an acceptable radial spectral steepening. In particular, at the $90 \%$ confidence level the values of the parameters are: $s=2 \pm 0.25, B_{\mathrm{c}} \sim 0.9 \div 1.2 \mu \mathrm{G}, l_{\mathrm{c}} \sim 15 \div 25 \mathrm{pc}$, $l_{\mathrm{c}}$ increasing with increasing the strength of the magnetic field $B_{\mathrm{c}}$. For these parameters, from Eq. (9), one obtains that the break energy at the cooling radius is $\gamma_{\mathrm{b}} \simeq 1500$. For one set of the parameters which best reproduces all the observational contraints we show in Figs. 4, 5 and 6 the fits to the surface brightness profile, total spectrum and radial spectral steepening.

For $0 \lesssim s \lesssim 1.5$ the space of the parameters reproducing the surface brightness profile is partially consistent with that producing an acceptable total synchrotron spectrum, but the derived spectral steepening is too strong. It is worth noticing that for $s$ approaching 0 the parameter limits obtained for the surface brightness profile and total synchrotron spectrum are not compatible, and, therefore, a constant relativistic energy density inside the cooling flow does not appear to agree with the observations. This means that the radial distribution of the number density of the electrons before re-acceleration may not be constant. For $s \lesssim 0$ and $s \gtrsim 2.5$ all the observational constraints cannot be reproduced by the same set of parameters.

On the other hand, our model results, assuming Soker \& Sarazin's case of radial field, are not compatible with the observations. In fact, we can not reproduce the spectral steepening for the same values of the parameters which produce the observed radio surface brightness profile and total synchrotron spectrum (Fig. 6). The rapid decrease of the magnetic field towards the outer regions in Soker \& Sarazin's case selects the relativistic electrons emitting between 327-609 $\mathrm{MHz}$ at progressively higher energies, causing a too rapid steepening of the synchrotron spectrum.

\section{Discussion and conclusions}

We have shown that the main properties of the radio mini-halo in the Perseus cluster can be accounted for by the synchrotron radiation from relic relativistic electrons in the cluster, which are efficiently re-accelerated by MHD Fermi type mechanisms due to the compression of the cluster magnetic field in the cooling flow region (see Sect. 3). Under the assumption of an efficient MHD turbulence with $\delta B / B \sim 1$ and spherical symmetry, our model has only 3 free parameters, namely the magnetic field intensity $B_{\mathrm{c}}$ and the turbulence scale $l_{\mathrm{c}}$ at the cooling

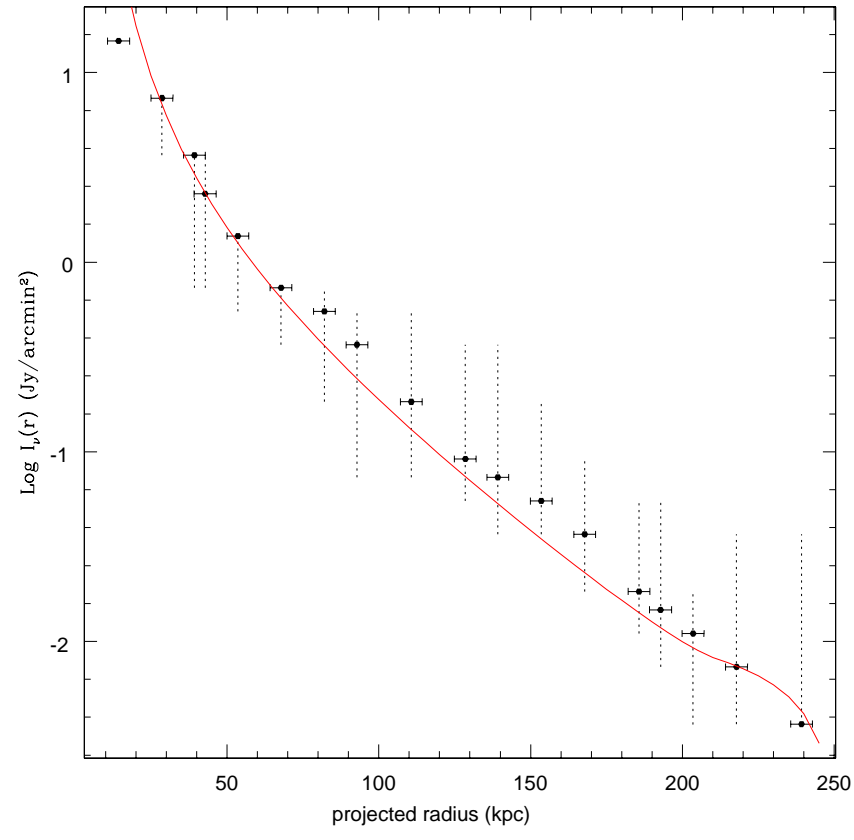

Fig. 4. Fit to the surface brightness profile obtained in the case of isotropic field with the following set of parameters: $B_{\mathrm{c}}=$ $1.2 \mu \mathrm{G}, l_{\mathrm{c}}=25 \mathrm{pc}, s=2.1$. Vertical errorbars represent the deviations from the spherical symmetry of the diffuse radio emission in other directions in the cluster with respect to the one we have considered.

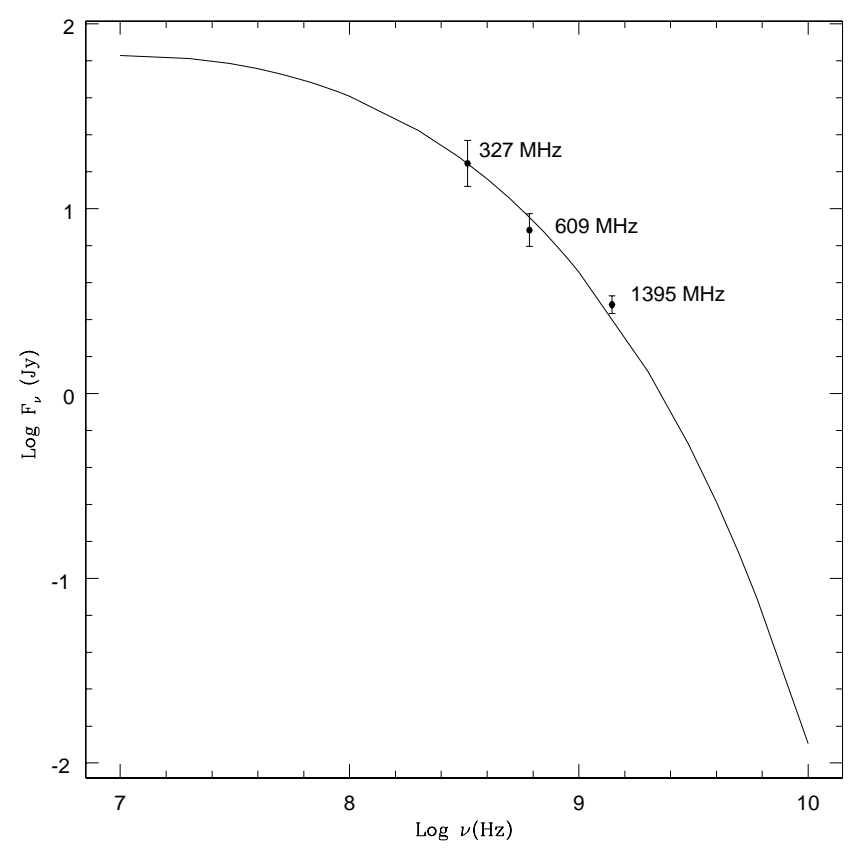

Fig. 5. Solid line: fit to the total spectrum obtained with the same set of parameters of Fig. 4. The radio data are taken from Sijbring (1993).

radius and the radial dependence of the relativistic electron energy density (parameterized as a power law of exponent $-s)$. One important conclusion we have reached is that, in our model, an isotropic field compression appears to well reproduce the observed surface brightness profile and total synchrotron spectrum along with the radial spectral steepening. The values of the parameters derived 


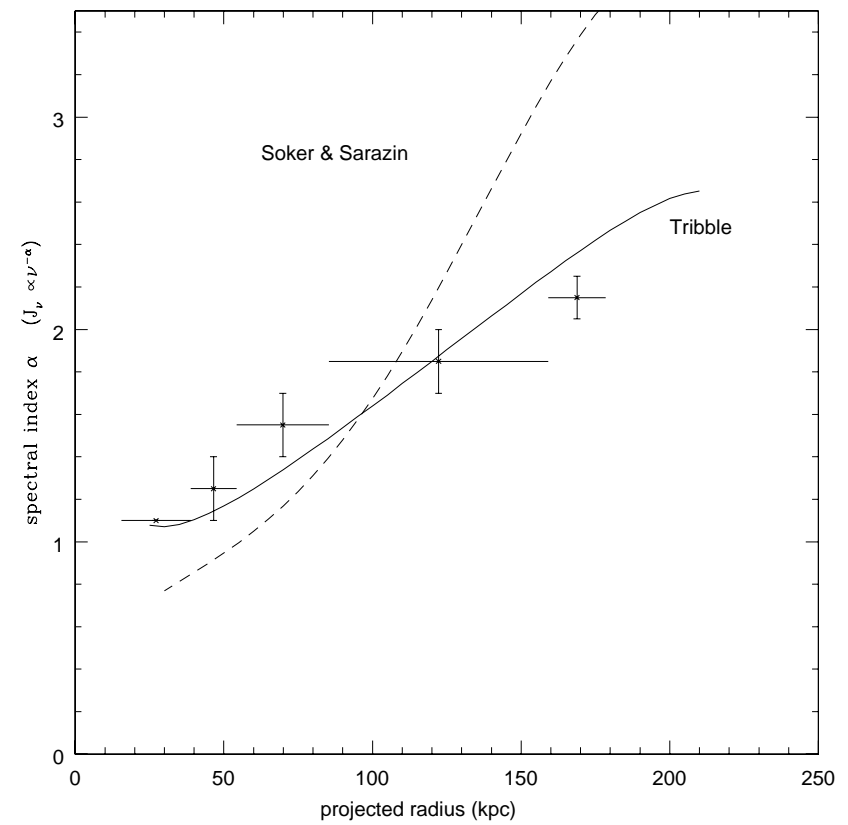

Fig. 6. Solid line: fit to the spectral steepening obtained with the same set of parameters of Fig. 4. The data are taken from spectral index distribution map of Sijbring (1993). Dashed line: predicted spectral steepening in the case of radial field, for the values of parameters which match the observed brightness profile and total spectrum.

by fitting all the observational constraints are: $s=2 \pm 0.25$, $B_{\mathrm{c}} \sim 0.9 \div 1.2 \mu \mathrm{G}, l_{\mathrm{c}} \sim 15 \div 25 \mathrm{pc}, l_{\mathrm{c}}$ increasing with increasing the strength of the magnetic field.

The fact that we can fit the spectral steepening for the same values of the parameters which provide a radio surface brightness profile and a total synchrotron spectrum in agreement with the observations (see Fig. 6) demonstrates the internal consistency of the model.

On the other hand, Soker \& Sarazin's arguments leading to a prevailing radial field in the cooling flow do not appear applicable to the mini-halo in the Perseus cluster, as it is not possible to reproduce the radial spectral steepening for the same set of parameters reproducing the brightness profile and total radio spectrum.

Our results showing the goodness of Tribble's model in reproducing all the observational constraints are consistent with the physical conditions prevailing in the cooling flow region of the Perseus cluster: the turbulent velocity $\left(v_{\mathrm{T}}=v_{\mathrm{A}} \sim 60 \mathrm{~km} \mathrm{~s}^{-1}\right.$ at the cooling radius) is greater than the mean inflow velocity $\left(v_{\mathrm{F}} \sim 20 \mathrm{~km} \mathrm{~s}^{-1}\right.$ at the cooling radius), and so we expect an isotropic compression of the field (see Sect. 2.1). Evidence in favour of Tribble's model in a number of cooling flows is also provided by Faraday rotation measurements (Taylor \& Perley 1993; Ge \& Owen 1993; Taylor et al. 1990; Perley \& Taylor 1991).

The range of values obtained for $B_{\mathrm{c}}$ is consistent with the measurements of magnetic fields diffused in the ICM (see Carilli \& Taylor 2002 and references therein).

The energetics associated with the re-accelerated electron population in the cooling flow region, $\sim 10^{59} \mathrm{erg}$, is smaller but not far from that required by detailed modeling for the electrons in the Coma radio halo $\left(\sim 5 \times 10^{59} \mathrm{erg}\right.$, case $c$ with $f=0.02$ in Brunetti et al. 2001). The number of relativistic electrons in the cooling flow region is $\sim 10^{62}$. This is comparable to the number of the electrons in a typical radiogalaxy and may suggest an important role of the AGNs (and possibly of the central AGN) in the injecton of the electron relic population.

Concerning the characteristic turbulence distance scale, a possible check for our model is whether or not the obtained values of $l_{\mathrm{c}}$ could be expected by some physical considerations on the turbulence in the ICM. In particular, assuming that the energy spectrum of Alfvén waves is a power law of the frequency $\omega,\left(\propto \omega^{-\nu}, \nu>1\right.$, Lacombe 1977), we examine the possible value of the minimum frequency $\omega_{\min }$, i.e. the wavelength $L=2 \pi v_{\mathrm{A}} / \omega_{\min }=$ $2 \pi / k_{\min }$ which carries most of the turbulent energy. Tsytovich (1972) suggests that the minimum frequency of an Alfvén wave spectrum is the collisional frequency of thermal protons, so that $k_{\text {min }} \sim k_{\mathrm{pp}} \equiv 2 \pi \nu_{\mathrm{pp}} / v_{\mathrm{A}}$, where $\nu_{\mathrm{pp}}=3 n_{\mathrm{p}} / T_{\mathrm{p}}^{3 / 2}$. For typical values of $n_{\mathrm{p}}$ and $T_{\mathrm{p}}$ in clusters of galaxies $\left(n_{\mathrm{p}} \sim 10^{-3} \mathrm{~cm}^{-3}, T_{\mathrm{p}} \sim 10^{7} \div 10^{8} \mathrm{~K}\right)$, we obtain $L \sim 2 \times 10^{1 \div 2} \mathrm{pc}$, consistent with the value of $l_{\mathrm{c}}$ obtained by our model.

In the framework of our model, the possibility of giving rise to an observable radio mini-halo in a cooling flow region is related to several physical parameters. We have found that the electron spectrum near the cooling radius has a break energy which, for the values of parameters derived by fitting all the observational constraints, is approximately $\gamma_{\mathrm{b}} \sim 1500$. This value corresponds to the break energy of the relativistic electrons outside the cooling flow region, where no significant diffuse radio emission is detected. As the typical life-time of $\gamma \sim 1500$ electrons is $\sim 1.6 \times 10^{9} \mathrm{yr}$, much less than the Hubble time, to balance the radiative losses of these electrons large-scale turbulence in the cluster volume is required. This scenario is similar to that of the classical cluster radio halos where, however, as the average magnetic field strength is considerably smaller than that in the cooling flow, higher energy electrons $\left(\gamma_{\mathrm{b}} \sim 10^{4}\right)$ are required to emit in the radio band so that the turbulence efficiency would have to be correspondingly greater. In addition, in Perseus the turbulence can not be too high in order to avoid the disruption of the cooling flow. Based on these considerations, we suggest that the physical conditions of the ICM in a cooling flow cluster, which also shows a radio mini-halo, are intermediate between those which lead to the formation of "standard" extended cluster radio halos and those holding in cooling flows without radio halos. This could explain - at least qualitatively - the rarity of radio minihalos found in cooling flow clusters. The energetics of the turbulence plays the main part in discriminating between these two opposite situations. A turbulence with a high enough energy density will be efficient at re-accelerating the relativistic electrons: depending on the length scale of the turbulence, these electrons can produce a diffuse 
synchrotron emission either in the form of extended radio halo or radio mini-halo. On the other hand, if the energy density of the turbulence is too low, there will be no efficient re-acceleration of the electrons and consequently no formation of diffuse radio emission, independently of the turbulence scale. Here we stress that the Perseus cluster may represent a borderline case: in fact there is evidence for a relatively recent merger event (Schwarz et al. 1992; Sijbring 1993; Dupke \& Bregman 2001), which may have left a fossil turbulence still quite energetic, with a typical scale just above the limit for activating a radio halo, but not so irrelevant to produce no re-acceleration at all.

As a further step, we can set a quantitative constraint on the large-scale turbulence imposing that the re-acceleration time scale well outside the cooling radius be more than $4 \div 5 \times 10^{8} \mathrm{yr}$, as derived by models on the formation of extended radio halos in clusters (Brunetti et al. 2001). If this condition is verified then an extended radio halo will not be formed. Assuming MHD Alfvénic acceleration, we obtain $l_{\mathrm{c}}(\mathrm{pc}) \gtrsim 10 B_{\mathrm{c}}^{2}(\mu \mathrm{G}) /\left(n_{\mathrm{c}} / 10^{-3}\right)$, which represents the lower limit on the large-scale turbulence in order to avoid the formation of a radio halo. We notice that the value of the parameters obtained by our model for the Perseus cluster are relatively close (within a factor $\sim 2$ ) to this limit.

High resolution $\mathrm{X}$-ray studies with XMM-Newton have recently shown that the soft X-ray spectra of several cooling flow clusters of galaxies are inconsistent with standard cooling flow models as the gas cools down to about 2-3 KeV, but not at lower temperatures (Peterson et al. 2001; Kaastra et al. 2001; Tamura et al. 2001; Molendi \& Pizzolato 2001). How to reconcile cooling flow models with these observations is still matter of debate (e.g. Fabian et al. 2001 and references therein), but it should be stressed that the compression of the thermal plasma and of the magnetic field within the cooling region is well ascertained. As a consequence, even if the cooling flow is somewhere close to the central galaxy stopped, the basic results of our model remain unchanged: once the turbulence has been energyzed by the flow, it takes a time of order of a few Gyr for it to be dissipated. During this time, turbulence continues to re-accelerate the electrons and determines the radio properties of the mini-halo.

Acknowledgements. We woud like to thank Luigina Feretti for useful discussion. In particular, we would like to thank the referee Vahé Petrosian for very helpful suggestions which improved the presentation of this paper. This work is partially supported by the Italian Ministry for University and Research (MURST) under grant 99GITTPGR Prog. Giov. Ricerc. E.F. 1999 .

\section{References}

Baum, S. A., \& O'Dea, C. P. 1991, MNRAS, 250, 737

Blasi, P., \& Colafrancesco, S. 1999, Astroparticle Phys., 12, 169

Böringer, H., Voges, W., Fabian, A. C., Edge, A. C., \& Neumann, D. N. 1993, MNRAS, 264, L25
Borovsky, J. E., \& Eilek, J. A. 1986, ApJ, 308, 929

Brunetti, G., Setti, G., Feretti, L., \& Giovannini, G. 2001 MNRAS, 320, 365

Burns, J. O., Sulkanen, M. E., Gisler, G. R., \& Perley, R. A. 1992, ApJ, 388, L49

Carilli, C. L., \& Taylor, G. B. 2002, ARA\&A, 40, in press

Dennison, B. 1980, ApJ, 239, L93

Dolag, K., \& Enßlin, T. A. 2000, A\&A, 362, 151

Dupke, R. A., \& Bregman, J. N. 2001, ApJ, 547, 705

Edge, A. C., Stewart, G. C., \& Fabian, A. C. 1992, MNRAS, 258,177

Eilek, J. A., \& Hughes, P. A. 1991, in Beams and Jets in Astrophysics, ed. P. A. Hughes (Cambridge University Press)

Ettori, S., Fabian, A. C., \& White, D. A. 1998, MNRAS, 300, 837

Fabian, A. C., Nulsen, P. E. J., \& Canizares, C. R. 1984, Nature, 310, 733

Fabian, A. C. 1994, ARA\&A, 32, 277

Fabian, A. C., Sanders, J. S., Ettori, S., et al. 2000, MNRAS, $318, \mathrm{~L} 65$

Fabian, A. C., Mushotzky, R. F., Nulsen, P. E. J., \& Peterson, J. R. 2001, MNRAS, 321, L20

Feretti, L. 2000 [astro-ph/0006379]

Feretti, L., \& Giovannini, G. 1996, IAUS, 175, 333

Ge, J. P., \& Owen, F. N. 1993, AJ, 105, 778

Ginzburg, V. L., \& Syrovatskii, S. I. 1965, ARA\&A, 3, 297

Govoni, F., Enßlin, T. A., Feretti, L., \& Giovannini, G. 2001, A\&A, 369, 441

Hamilton, R. J., \& Petrosian, V. 1992, ApJ, 398, 350

Kaastra, J. S., Ferrigno, C., Tamura, T., et al. 2001, A\&A, 365, L99

Kardashev, N. S. 1962, Sov. Astron., - AJ, 6, 3

Lacombe, C. 1977, A\&A, 54, 1

Liang, H., Hunstead, R. W., Birkinshaw, M, \& Andreani, P. 2000, ApJ, 544, 686

Melrose, D. B. 1980, Plasma Astrophysics: Nonthermal Processes in Diffuse Magnetized Plasmas (Gordon and Breach)

Molendi, S., \& Pizzolato, F. 2001, ApJ, 560, 194

Owen, F. N., Eilek, J., \& Kassim, N. E. 2000, ApJ, 543, 611

Perley, R. A., \& Taylor, G. B. 1991, AJ, 101, 1623

Peterson, J. R., Paerels, F. B. S., Kaastra, J. S., et al. 2001, A\&A, 365, L104

Petrosian, V. 2001, ApJ, 557, 560

Rizza, E., Loken, C., Bliton, M., Roettiger, K., \& Burns, J. O. 2000, AJ, 119, 21

Sarazin, C. L. 1999, ApJ, 520, 529

Sarazin, C. L. 2001, to appear in Merging Processes in Clusters of Galaxies, ed. L. Feretti, I. M. Gioia, \& G. Giovannini (Dordrecht: Kluwer), in press [astro-ph/0105418]

Schwarz, R. A., Edge, A. C., Voges, W., et al. 1992, A\&A, 256, L11

Sijbring, D. 1993, A Radio Continuum and HI Line Study of the Perseus Cluster, Ph.D. Thesis, Groningen

Soker, N., \& Sarazin, C. L. 1990, ApJ, 348, 73

Tamura, T., Kaastra, J. S., Peterson, J. R., et al. 2001, A\&A, 365, L87

Taylor, G. B., Perley, R. A., Inoue, M., et al. 1990, ApJ, 360, 41

Taylor, G. B., \& Perley, R. A. 1993, ApJ, 416, 554

Tribble, P. C. 1993, MNRAS, 263, 31

Tsytovich, V. N. 1972, An Introduction to the Theory of Plasma Turbulence (Pergamon Press) 